\title{
Perforated Appendicitis Within a Morgagni Hernia: A Laparoscopic Repair
}

\author{
Margaret E. Clark, MD, Benjamin D. Tabak, MD, Andrew T. Schlussel, DO, \\ Jeffery M. Meadows, MD, John D. Andersen, BS, MA, Mary J. Edwards, MD, \\ Stanley M. Zagorski, MD \\ Department of Surgery, Tripler Army Medical Center, Honolulu, HI, USA (Drs. Clark, Tabak, Schlussel, Zagorski, \\ Edwards). \\ Department of Radiology, Tripler Army Medical Center, Honolulu, HI, USA (Dr. Meadows). \\ Arizona College of Osteopathic Medicine, Midwestern University, Glendale, AZ, USA (Dr. Andersen).
}

\begin{abstract}
Laparoscopic repair of a Morgagni hernia is a well-described technique in children or adults who present well beyond the neonatal period. However, it is still most often being repaired by an open approach. This repair can be more challenging when a concomitant infectious process is present, which in this case was a perforated appendicitis. A 22-year-old man presented with typical appendicitis but was found to have a perforated appendicitis contained within a congenital diaphragmatic hernia. We demonstrate the safety and efficacy of using laparoscopy to repair a Morgagni hernia, in the setting of a contaminated field, with primary suture repair. Our patient was able to have both his appendectomy and his Morgagni hernia repair with a single operation, and there was no recurrence of the diaphragmatic defect.
\end{abstract}

Key Words: Morgagni hernia, Congenital diaphragmatic hernia, Appendicitis in a hernia, Laparoscopic hernia repair.

\footnotetext{
Citation Clark ME, Tabak BD, Schlussel AT, Meadows JM, Andersen JD, Edwards MJ, Zagorski SM. Perforated appendicitis within a morgagni hernia: a laparoscopic repair. CRSLS e2014.00075. DOI: 10.4293/CRSLS.2014.00075.

Copyright (C) 2014 SLS This is an open-access article distributed under the terms of the Creative Commons Attribution-Noncommercial-ShareAlike 3.0 Unported license, which permits unrestricted noncommercial use, distribution, and reproduction in any medium, provided the original author and source are credited.

The opinions expressed in the article are solely those of the authors and do not represent the views or official policies of the US Army, Department of Defense, or US Government.

Disclosure: The authors report no conflict of interest-financial, personal, or professional-concerning the preparation of this article. There is no off-label use described in this article.

Address correspondence to: Stanley M. Zagorski, MD, Department of General Surgery, Tripler Army Medical Center, One Jarrett White Road, Honolulu, HI 96859, USA. Tel (808) 433-3447, Fax: (808) 433-6539, E-mail:zagorsks@hotmail.com
}

\section{INTRODUCTION}

Morgagni hernia is a rare congenital defect found in the anterior aspect of the diaphragm, accounting for only 3\% of all diaphragmatic hernias. The clinical presentation is uncommon in adulthood. In our patient, his defect was discovered when perforated appendicitis developed inside the thoracic cavity. Both conditions were repaired simultaneously through a laparoscopic approach.

\section{CASE REPORT}

A 22-year-old man presented to the emergency department with a 1-day history of sharp and cramping perium- bilical pain that migrated to the right lower quadrant. Nausea and emesis developed after the onset of pain, followed by fever. The patient denied any respiratory symptoms. His medical and surgical history was unremarkable.

Physical examination was significant for a marfanoid body habitus, pectus excavatum, and fever. The patient's breath sounds were diminished on the right. He had a scaphoid abdomen with right lower quadrant tenderness, predominantly over the McBurney point, but no Rovsing sign or diffuse peritonitis. Laboratory examinations showed leukocytosis $(18300 / \mu \mathrm{L})$ with a left shift, with $82.2 \%$ granulocytes. Abdominal computed tomography (CT) (Figure 1) 


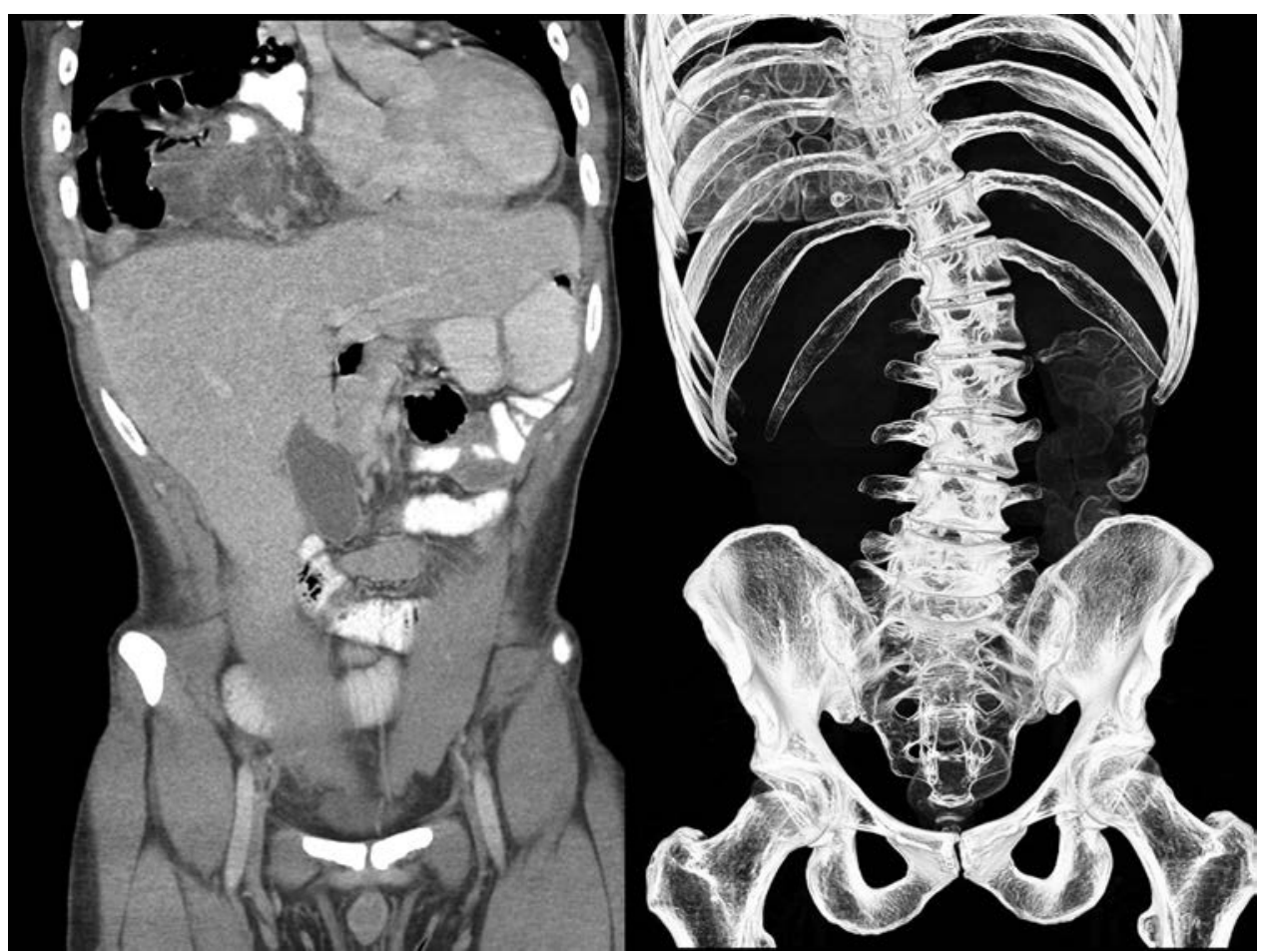

Figure 1. Coronal CT scan of abdomen and pelvis with intravenous and oral contrast (left) and corresponding volume-rendered 3-dimensional image accentuating tissues and structures with higher densities, such as contrast, calcium, and bone (right).

showed not only that the patient had bowel above the liver but also that part of the colon was above the diaphragm in the lower right hemithorax. There was free fluid overlying the liver and extending into the right paracolic gutter of the lower abdomen, likely accounting for the location of his pain and tenderness. There appeared to be a defect in the anterior right hemidiaphragm, through which a mesenteric pedicle was seen connecting the herniated bowel with the abdominal cavity. The herniation was believed to be a congenital Morgagni hernia, given the patient's age, its anterior location, and lack of trauma in the patient's history. Closer evaluation of the hernia sac showed a contained inflammatory process centrally, with a calcification believed to be an appendicolith. A few punctate foci of extraluminal gas, causing concern regarding a perforation, were also seen. Although the appendix was not clearly identified, it was believed to be within this collection because the cecum was clearly within the hernia sac. The patient was taken to the operating room for diagnostic laparoscopy.

Intraoperatively, a $12-\mathrm{mm}$ port was placed at the umbilicus with two 5-mm working ports, one in the left upper quadrant and one in the right upper quadrant. It was confirmed that the patient had an anterior, retrosternal diaphragmatic hernia with a perforated appendix within the chest (Figure 2A). The herniated small bowel and large bowel were reduced from the chest back into the abdomen by providing gentle traction after thin adhesions from the mesentery to the diaphragm were taken down with electrocautery. A periappendiceal abscess was identified and drained, and a standard laparoscopic appendectomy was performed once the appendix was located in its standard intraabdominal position. This was performed with the addition of a suprapubic and left lower quadrant port, and the specimen was removed with an endoscopic retrieval bag. The entire abdomen was suctioned and irrigated before attention was turned back toward the diaphragmatic defect. Intraoperatively, the ligament of Treitz was inspected and found to be normal.

The Morgagni hernia defect was approximately $7 \mathrm{~cm}$ in diameter. This was repaired by passing multiple nonabsorbable sutures through the abdominal wall and diaphragmatic defect and tying them extracorporeally. Additional sutures were placed intracorporeally to approximate the diaphragm to the posterior rectus sheath. The hernia sac was left in situ. The completed repair was under minimal to no tension (Figure 2B). 

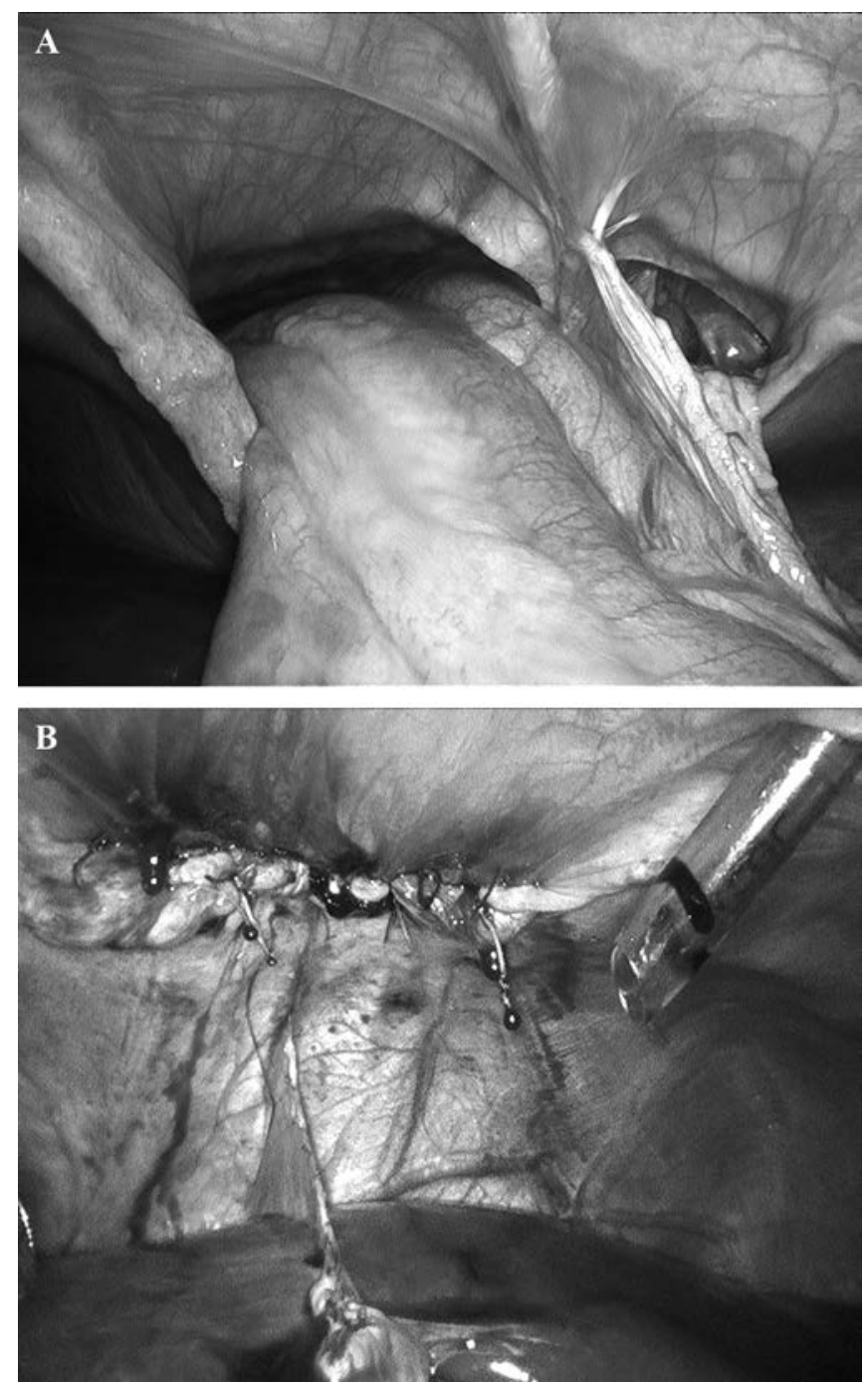

Figure 2. A, Following the ascending colon up into the diaphragmatic defect, where the perforated appendix was found. $\mathbf{B}$, Laparoscopic closure of Morgagni defect.

The patient recovered well and was discharged home on postoperative day 3 . He returned 2 weeks later with a pelvic abscess, which was managed with percutaneous drainage and antibiotics. CT scan to evaluate for abscess showed an intact diaphragmatic repair. At 9 months postoperatively, he is symptom free and without evidence of recurrence of his hernia; he has returned to military service.

\section{DISCUSSION}

In 1769, Giovanni Morgagni first described a rare anterior, retrosternal defect in the diaphragm., ${ }^{1,2}$ Also referred to as
Larry hernia, retrosternal hernia, or parasternal hernia, the defect usually has a sac and likely results from lack of ingrowth of the cervical myotomes. It is the result of failure of fusion of the fibrotendinous portion of the pars tendinalis arising from the costochondral arches with the fibrotendinous portion of the pars sternalis. This creates a space between the sternal and costal origin of the diaphragm. ${ }^{1}$ These hernias typically occur on the right side. The condition has been associated with multiple syndromes and congenital defects, including connective tissue disorders and chest wall deformities. A hereditary link has been hypothesized but never proven. In one study of 16 pediatric patients with Morgagni hernias, $75 \%$ had associated congenital anomalies and 3 patients had pectus carinatum. ${ }^{3}$

Late-presenting (after the newborn period) diaphragmatic hernias are rare in developed countries because of prenatal and postnatal diagnostic imaging. ${ }^{4}$ In a review of 30 patients with presentations in childhood (aged 12 months to 15 years), Marhuenda et al. ${ }^{5}$ suggested that patients may present with gastrointestinal or respiratory symptoms or may be asymptomatic. If symptomatic, patients often present with shortness of breath, ileus, food intolerance, reflux, nausea, vomiting, cramping, or distension. They have also been reported to present with recurrent pneumonia. ${ }^{2}$ However, repair is recommended regardless of presentation because of the potential complications, including gastric volvulus, intestinal obstruction, and perforation. In their report they identified laparoscopy as a safe approach. ${ }^{5}$

The diagnosis of a Morgagni hernia may be suggested on chest radiographs, which may show a bowel gas pattern in the chest. Abnormal findings are usually followed by CT scans of the chest and abdomen, which may show contrast-filled bowel in the chest. A diagnosis of diaphragmatic hernia may be suggested by fine curvilinear densities within the mass in the chest, which represent vessels in the omentum, the most commonly herniated organ. Three-dimensional imaging using helical CT has also been suggested as useful because no oral contrast is required. ${ }^{6}$ Finally, if no abdominal viscera are located in the chest, magnetic resonance imaging may be able to distinguish between a chest process and a process that originates from within the abdomen.

Only a few additional cases of intrathoracic appendicitis have been reported in the literature, and none clearly describe a congenital Morgagni hernia or a laparoscopic repair. These reported cases have typically presented with 
chest pain, which would be expected given that the inflammatory process is intrathoracic. ${ }^{7-10}$

There has been one other case of intrathoracic appendicitis presenting with classic signs and symptoms of appendicitis. Barakat and Vickers ${ }^{11}$ reported a case wherein a woman with marfanoid features was found to have intrathoracic appendicitis. The case was initially approached through a standard right iliac fossa incision. When the appendix could not be found, exploratory laparoscopy was performed. The colon was identified to be herniated into the chest through a defect in the right diaphragm beneath the right lobe of the liver. The procedure was abandoned so that a CT scan could be performed to define the anatomy. The appendix was later reduced into the abdomen, again similar to other cases, through laparotomy, and the hernia was not repaired.

Given our patient's presentation, there would be little reason to suspect an abnormal location of the appendix. It would have been reasonable to proceed with laparoscopic appendectomy without CT scan. The emergency department obtained the CT scan before the surgical consultation. In this case, having the scan proved to be beneficial given that it allowed us to perform better preoperative planning and still allowed us to perform laparoscopic appendectomy but also repair the patient's Congenital diaphragmatic hernia using the same laparoscopic approach and with only one operation.

Morgagni hernias that present in adulthood may be a result of new (traumatic) defects or congenital defects that have enlarged over time due to increased intra-abdominal pressure (pregnancy, trauma, obesity, chronic constipation, and chronic cough). In a review of 298 adult patients treated from 1951 to 2006, thoracotomy was the most widely used approach for repair of Morgagni hernias. ${ }^{4}$ In another more recent study, diaphragmatic hernias between 1999 and 2008 still were mostly repaired through open techniques despite advances in laparoscopic surgery. ${ }^{12}$ The abdominal approach, whether laparoscopic or open, allows for easier reduction of hernia contents and easier evaluation and repair of intra-abdominal pathology. The first review identified 46 laparoscopic repairs of adult Morgagni hernias presented in the literature. During laparoscopic repair in adults, the defect was usually repaired with mesh (64\%) and the hernia sac was not removed (69\%). Surgeons cited longer operative time, postoperative pneumothorax, and fatal pneumopericardium as reasons for not removing the sac. ${ }^{13}$ Thoman et al. ${ }^{14}$ recommended sewing prosthetic mesh to cover defects larger than 20 to $30 \mathrm{~cm}^{2} .13,15$ Other authors recommended using mesh repairs in all cases except those involving neonates. Many materials have been used, including polypropylene, expanded polytetrafluoroethylene, and bovine pericardium. Given our patient's concomitant infectious process, we chose to repair the hernia defect primarily without mesh.

\section{CONCLUSION}

A Morgagni hernia is a congenital defect that may obscure the diagnosis of common intra-abdominal pathology, particularly appendicitis. Laparoscopic repair of these defects is a well-described technique in children or adults who present well beyond the neonatal period. However, the presentation of appendicitis within the hernia sac creates a contaminated field, which may make conventional repair with prosthetic mesh inappropriate. In these cases a suture repair may be used if the defect can be closed without excessive tension. If the defect cannot be closed immediately, repair of the hernia may be delayed until the acute infection has resolved, and a mesh repair can be performed. Alternatively, a biologic mesh could be considered.

\section{References:}

1. Nasr A, Fecteau A. Foramen of Morgagni hernia: presentation and treatment. Thorac Surg Clin. 2009;19:463-468.

2. Gedik E, Tuncer MC, Onat S, Avci A, Tacyildiz I, Bac B. A review of Morgagni and Bochdalek hernias in adults. Folia Morphol (Warsz). 2011;70:5-12.

3. Cigdem MK, Onen A, Okur H, Otcu S. Associated malformations in Morgagni hernia. Pediatr Surg Int. 2007;23:11011103.

4. Horton JD, Hofmann LJ, Hetz SP. Presentation and management of Morgagni hernias in adults: a review of 298 cases. Surg Endosc. 2008;22:1413-1420.

5. Marhuenda C, Gvillén G, Sánchez B, Urbistondo A, Barceló C. Endoscopic repair of late presenting Morgagni and Bochdalek Hernia in children: Case Report and review of the literature. $J$ Laparoendosc Adv Surg Tech A. 2009;19:S95-101.

6. Yamashita K, Tsunoda T. Three-dimensional computer images of Morgagni hernia. Am J Surg. 2004;187(1):109-110.

7. Schellhaas E, Döbler O, Kroesen AJ, Buhr HJ, Hotz HG. Gangrenous intrathoracic appendicitis, a rare cause of rightsided chest pain: report of a case. Surg Today. 2010;40:874-877.

8. Kshirsagar AY, Bansal SS, Somnath SR, Prabhu AN, Dhulkhed V, Nikumbh DB. Acute appendicitis presenting as chest pain. Int J Surg Case Rep. 2012;3:128-130. 
9. Gürthes N, Gürthes N. Perforating appendicitis within a diaphragmatic hernia: a case report. Z Kinderchir. 1986;41: $306-307$.

10. Zerin JM. Intrathoracic appendicitis in a ten-year-old girl. Roentgenologic clinical pathologic case. Invest Radiol. 1990; 25(10):1162-1164.

11. Barakat MJ, Vickers JH. Necrotic gangrenous intrathoracic appendix in a marfanoid adult patient: a case report. BMC Surg. 2005;5:4.

12. Paul S, Nasar A, Port JL, et al. Comparative analysis of diaphragmatic hernia repair outcomes using the nationwide inpatient sample database. Arch Surg 2012;147(7):607-612.
13. Palanivelu C, Rangarajan M, Rajapandian S, Amar V, Parthasarathi R. Laparoscopic repair of adult diaphragmatic hernias and eventration with primary sutured closure and prosthetic reinforcement: a retrospective study. Surg Endosc. 2009;23:978-985.

14. Thoman DS, Hui T, Phillips EH. Laparoscopic diaphragmatic hernia repair. Surg Endosc. 2002;16:1345-1349.

15. Schumacher L, Gilbert S. Congenital diaphragmatic hernia in the adult. Thorac Surg Clin. 2009;19:469-472. 